\title{
8
}
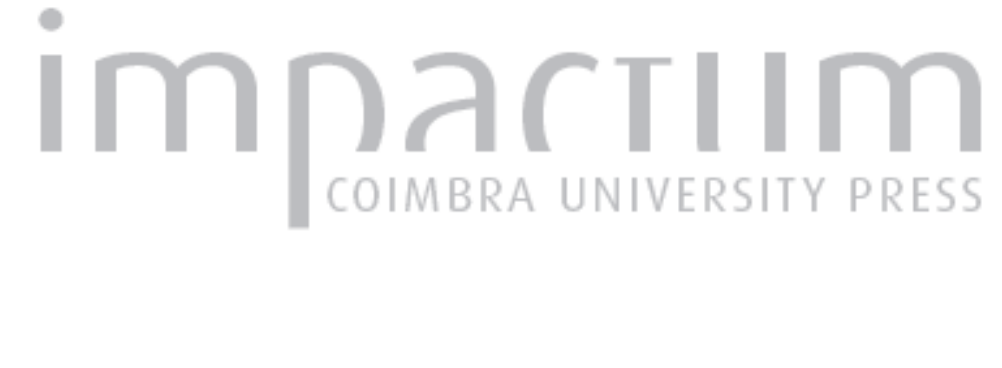

\section{Avaliação do risco para a navegação utilizando o sistema Guiomar: o caso do porto} de Sines

\author{
Autor(es): $\quad$ Neves, Diogo Ruben; Fortes, Conceição Juana; Santos, João Alfredo; \\ Reis, Maria Teresa; Rodrigues, Sara \\ Publicado por: Associação Portuguesa de Riscos, Prevenção e Segurança \\ URL \\ persistente: \\ URI:http://hdl.handle.net/10316.2/36050 \\ DOI: \\ DOI:http://dx.doi.org/10.14195/1647-7723_18_7 \\ Accessed : $\quad$ 26-Apr-2023 14:00:49
}

A navegação consulta e descarregamento dos títulos inseridos nas Bibliotecas Digitais UC Digitalis, UC Pombalina e UC Impactum, pressupõem a aceitação plena e sem reservas dos Termos e Condições de Uso destas Bibliotecas Digitais, disponíveis em https://digitalis.uc.pt/pt-pt/termos.

Conforme exposto nos referidos Termos e Condições de Uso, o descarregamento de títulos de acesso restrito requer uma licença válida de autorização devendo o utilizador aceder ao(s) documento(s) a partir de um endereço de IP da instituição detentora da supramencionada licença.

Ao utilizador é apenas permitido o descarregamento para uso pessoal, pelo que o emprego do(s) título(s) descarregado(s) para outro fim, designadamente comercial, carece de autorização do respetivo autor ou editor da obra.

Na medida em que todas as obras da UC Digitalis se encontram protegidas pelo Código do Direito de Autor e Direitos Conexos e demais legislação aplicável, toda a cópia, parcial ou total, deste documento, nos casos em que é legalmente admitida, deverá conter ou fazer-se acompanhar por este aviso.

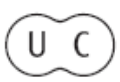




\section{territorium}

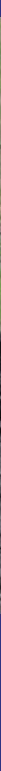

\section{Afirmar as Ciências Cindínicas}

Revista da Associação Portuguesa de Riscos, Prevenção e Segurança 
Diogo Ruben Neves dneves@lnec.pt; Conceição Juana Fortes jfortes@lnec.pt; João Alfredo Santos jasantos@lnec. pt; Maria Teresa Reis mtreis@lnec.pt e Sara Rodrigues srodrigues@lnec.pt

Núcleo de Portos e Estruturas Marítimas, Departamento de Hidráulica e Ambiente, Laboratório Nacional de Engenharia Civil

\section{RESUMO}

O presente trabalho descreve uma primeira aplicação ao porto de Sines do conjunto de procedimentos automáticos introduzidos no sistema integrado de modelação da agitação marítima, GUIOMAR, para avaliação do risco para navegação em zonas portuárias. O novo conjunto de procedimentos automáticos implementa uma metodologia de avaliação do risco baseada na amplitude da componente vertical do movimento de um navio ao longo da sua trajectória induzida pela agitação marítima incidente no navio.

Palavras chave: Avaliação de Risco: Navegação: Sistema de Informação Geográfica: Porto de Sines: GUIOMAR.

\section{RESUMEN}

Evaluación de riesgos para el tráfico marítimo utilizando GUIOMAR. El caso del puerto de Sines - El presente trabajo describe una primera aplicación al puerto de Sines del conjunto de procedimientos automáticos introducidos en el sistema integrado de modelación de oleaje, GUIOMAR, para evaluar los riesgos para el tráfico de navíos en puertos. Dicho conjunto implementa una evaluación de riesgos basada en la altura de la componente vertical del movimiento de un navío a lo largo de su trayecto provocada por el oleaje incidente en el navío.

Palabras clave: Evaluación de riesgos, Tráfico marítimo, Sistema de Información Geográfica, Puerto de Sines, GUIOMAR.

\section{RESUMÉ}

Evaluation des risques pour la navigation en utilisant le système GUIOMAR. Le cas du port de Sines - Ce travail décrit une première application au port de Sines de l'ensemble de procédures automatiques introduites au système intégré de modélisation des houles maritimes, GUIOMAR, pour évaluer les risques pour la navigation portuaire. Cet ensemble implémente une évaluation des risques basée à l'hauteur de la component verticale du mouvement d'un navire le long de sa route causé par les houles qui atteignent le navire.

Mots clé: Evaluation des risques, Navigation, Système d'information géographique, Port de Sines, GUIOMAR.

\section{ABSTRACT}

Risk Assessment for the navigation using the GUIOMAR system. The port of Sines study case - This paper describes a first approach on the risk assessment in port navigation using the GUIOMAR, an integrated system for port and coastal engineering modelling using a GIS software environment. A set of automatic procedures was designed to include a new methodology based on the amplitude of the wave-induced vertical movement of a ship along its trajectory. In this methodology, the risk in port navigation is assessed on the basis of a combination of the probability of exceedance of a pre-set threshold for the ship's vertical movements and its consequences. The port of Sines study case was used to test the new procedures.

Key words: Risk Assessment: Navigation: Geographical Information Systems: Port of Sines: GUIOMAR.

\footnotetext{
* O texto deste artigo corresponde à comunicação apresentada ao II Congresso Internacional de Riscos e VI Encontro Nacional, tendo sido submetido para revisão em 31-05-2010, tendo sido aceite para publicação em 18-07-2010.

Este artigo é parte integrante da Revista Territorium, n. ${ }^{\circ}$ 18, 2011, ${ }^{\circledR}$ RIscos, ISBN: 0872- 8941.
} 


\section{Introdução}

Os riscos associados à navegação no interior de portos são uma das principais preocupações para engenheiros navais e portuários. As consequências de situações indesejadas podem ser graves quando existe manifesta falta de segurança, nomeadamente no que se refere a perdas de bens materiais, de vidas e a consequências para o meio ambiente. 0 exposto justifica a importância crescente do desenvolvimento de metodologias capazes de avaliar o risco neste tipo de situações.

Atendendo à natureza dos portos, o cumprimento do acima proposto leva à necessidade de integrar dados de agitação marítima medidos na proximidade do porto com resultados de modelos numéricos de forma a simular um conjunto de cenários capaz de conter condições menos favoráveis à segurança do porto ou dos navios que o frequentam. Um melhor conhecimento das características da agitação marítima pode ser obtido utilizando modelos numéricos de propagação e deformação da agitação marítima que permitem a simulação de cenários reais.

A aplicação dos modelos numéricos é complexa e requer tempo, quer para a preparação dos dados de entrada, quer para a análise dos resultados obtidos, uma vez que os modelos numéricos conduzem a grandes quantidades de informação. Daí o interesse na utilização de Sistemas de Informação Geográfica (SIG) pois permitem uma análise espacial e temporal de informação correspondente a fenómenos naturais complexos através da amostra de tendências e padrões que são essenciais para qualquer processo rápido de tomada de decisão. Com efeito, os sistemas SIG têm a capacidade de facilmente produzir mapas de risco de acordo com procedimentos automáticos baseados em dados e resultados de modelos numéricos, facilitando assim o processo de tomada de decisão na gestão costeira e portuária.

Tem vindo a ser desenvolvido no LNEC um sistema integrado de modelação costeira e portuária, GUIOMAR (D.R.C.B. Neves et al., 2009A) com base no software comercial $\mathrm{ArcGis}^{\mathrm{TM}}$ e que permite a utilização de vários modelos numéricos de propagação de ondas num ambiente SIG. Este sistema foi criado para apoiar a tomada de decisão na gestão de processos característicos de zonas costeiras e portuárias.

O presente trabalho descreve a metodologia introduzida no sistema GUIOMAR para avaliar o risco para a navegação no interior de um porto recorrendo aos modelos numéricos de geração, propagação e dissipação da agitação marítima SWAN (N. Bools et al., 1999) e DREAMS (C.J.E.M. Fortes, 2002), e ao modelo numérico WAMIT (J.N. Newman e P.D. Sclavounos, 1988) para o cálculo dos movimentos do navio. Esta metodologia foi concretizada através de um novo módulo no sistema GUIOMAR que aplica os procedimentos de avaliação de risco de forma automática e possibilita ao utilizador a constituição de mapas de risco pré-formatados.

Além deste módulo, o trabalho apresenta também a aplicação do mesmo ao porto de Sines, uma das entradas marítimas mais importantes para Portugal e para a Europa, localizado na costa sudoeste de Portugal continental, $37^{\circ} 57^{\prime} \mathrm{N}$ e $08^{\circ} 53^{\prime} \mathrm{W}$. Este caso de estudo envolveu: (i) o cálculo do regime geral de agitação nos centros de gravidade dos troços da trajectória do navio no porto; (ii) o cálculo dos movimentos verticais de um ponto seleccionado do navio em cada um dos centros de gravidade; e (iii) o cálculo do grau de risco associado à excedência de um limiar pré-estabelecido para um determinado movimento vertical, a partir da análise quantitativa da probabilidade de ocorrência e da análise qualitativa do grau de consequências.

Nas secções seguintes, apresentam-se a metodologia de avaliação do risco, os novos desenvolvimentos ao sistema GUIOMAR com a respectiva implementação dos procedimentos automáticos de avaliação de risco e o caso de estudo aplicado ao porto de Sines.

\section{Metodologia de avaliação do risco}

A metodologia adoptada neste trabalho tem como base a definição de risco como o produto da probabilidade de ocorrência de um acontecimento adverso pelo custo das consequências desse acontecimento. No problema da navegação portuária, este trabalho indica que o acontecimento adverso é a amplitude do movimento vertical de um ponto do navio em manobra exceder um patamar prédefinido ao longo da sua viagem de entrada no porto de Sines.

Para calcular esta probabilidade de ocorrência basta considerar a distribuição das alturas dos movimentos verticais calculados para uma amostra significativa de estados de agitação num dos pontos da trajectoria. Embora não seja difícil estimar a probabilidade de ocorrência do acontecimento adverso, por uma questão de apresentação de resultados, é mais interessante atribuir um grau à mesma probabilidade de ocorrência, de acordo com o seu valor. No QUADRo I apresenta-se uma classificação preliminar adoptada para a probabilidade de ocorrência de um acontecimento adverso.

QUADRo I - Probabilidade de Ocorrência de movimentos verticais de um navio que excedem limiares pré-estabelecidos.

\begin{tabular}{|c|c|c|}
\hline \multirow{2}{*}{ Descrição } & $\begin{array}{c}\text { Probabilidade de Ocorrência } \\
\text { (Guia de Orientação) }\end{array}$ & Grau \\
\hline Improvável & $0-1 \%$ & 1 \\
\hline Raro & $1-10 \%$ & 2 \\
\hline Ocasional & $10-25 \%$ & 3 \\
\hline Provável & $25-50 \%$ & 4 \\
\hline Frequente & $>50 \%$ & 5 \\
\hline
\end{tabular}


Nesta metodologia de avaliação do risco, a tarefa mais complexa é a atribuição de custos às consequências do acontecimento adverso. Para facilitar a definição desses custos, algumas organizações produzem directivas que propõem a classificação do custo esperado de acordo com as consequências do acontecimento adverso para as pessoas, para o património relativo aos bens materiais do navio (tanto a nível do próprio navio, como da sua mercadoria), para o ambiente e o ecossistema em que está inserido o porto e para a gestão portuária a nível de contratos económicos e transacções comerciais. No Quadro II apresenta-se uma dessas classificações baseada nas directivas da NEW ZEALAND MARITIME SAFETY AUTHORITY, 2004.

Os valores do grau de Consequências foram atribuídos de forma a ser possível ter em conta a importância do risco no que diz respeito ao seu controle e prioritização. Por exemplo, é importante distinguir entre um evento com elevado grau de Probabilidade de Ocorrência mas com grau de Consequências baixo e um evento com baixo grau de Probabilidade de Ocorrência mas com grau de Consequências muito elevado.

Do produto do grau de Probabilidade de Ocorrência pelo grau de Consequências obtém-se o grau de Risco, QuADRo III. Embora a atribuição de um grau de Consequências torne a avaliação do risco mais qualitativa, esta utilização do grau de Risco tem a vantagem de ser mais compacta e de facilitar a comparação de soluções para a limitação da ocorrência do acontecimento adverso ou das suas consequências, bem como para a classificação da aceitabilidade do Risco obtido, QUADRo IV.

Quadro III - Grau de Risco.

\begin{tabular}{|c|c|c|c|c|c|c|}
\hline \multirow{2}{*}{\multicolumn{2}{|c|}{ GRAU DE RISCO }} & \multicolumn{5}{|c|}{ Consequências } \\
\hline & & 1 & 2 & 5 & 10 & 25 \\
\hline \multirow{5}{*}{$\begin{array}{l}\text { Probabilidade de } \\
\text { Ocorrência }\end{array}$} & 1 & 1 & 2 & 5 & 10 & 25 \\
\hline & 2 & 2 & 4 & 10 & 20 & 50 \\
\hline & 3 & 3 & 6 & 15 & 30 & 75 \\
\hline & 4 & 4 & 8 & 20 & 40 & 100 \\
\hline & 5 & 5 & 10 & 25 & 50 & 125 \\
\hline
\end{tabular}

Quadro IV - Avaliação da aceitabilidade do grau de Risco obtido.

\begin{tabular}{|c|c|c|}
\hline Grau & Descrição & Controlo do Risco (Guia de Orientação) \\
\hline $1-3$ & Insignificante & $\begin{array}{c}\text { Risco desprezável; não é necessário levar a cabo } \\
\text { medidas de controlo de risco. }\end{array}$ \\
\hline $4-10$ & Reduzido & $\begin{array}{c}\text { Risco que pode ser considerado aceitável/tolerável caso } \\
\text { se seleccione um conjunto de medidas para o seu } \\
\text { controlo. }\end{array}$ \\
\hline $15-30$ & Indesejável & $\begin{array}{c}\text { Risco que deve ser evitado se for razoável em termos } \\
\text { práticos; requer uma investigaçâo detalhada e análise de } \\
\text { custo-benefício; e essencial a monitorização. }\end{array}$ \\
\hline $40-125$ & Inaceitável & $\begin{array}{c}\text { Risco intolerável; tem que se proceder ao controlo do } \\
\text { risco (e.g. eliminar a origem dos riscos, alterar a } \\
\text { probabilidade de ocorrência e/ou as consequências, } \\
\text { transferir o risco, etc.). }\end{array}$ \\
\hline
\end{tabular}

Antes de qualquer avaliação de risco, primeiro é necessário determinar a altura do movimento vertical induzido pela agitação marítima ao longo da trajectória do navio. Para cada navio considerado e para cada um dos troços considerados para a trajectória do navio no interior do porto, a metodologia proposta neste trabalho consiste em três passos fundamentais (Fig. 1):

Quadro II - Consequências da ocorrência de movimentos verticais de um navio que excedem limiares pré-estabelecidos.

\begin{tabular}{|c|c|c|c|c|c|}
\hline \multirow{2}{*}{ Descrição } & \multicolumn{4}{|c|}{ Consequências (Guia de Orientação) } & \multirow{2}{*}{ Grau } \\
\hline & Pessoas & Património & Ambiente & Gestão Portuária & \\
\hline Insignificantes & $\begin{array}{c}\text { Existe a } \\
\text { possibilidade } \\
\text { de ferimentos } \\
\text { muito ligeiros }\end{array}$ & Insignificante & $\begin{array}{l}\text { Impacto ambiental } \\
\text { desprezável }\left(<10^{4} €\right)\end{array}$ & Insignificante $\left(<10^{4} €\right)$ & 1 \\
\hline Reduzidas & $\begin{array}{l}\text { Uma única } \\
\text { lesão ligeira }\end{array}$ & $10-10^{2} €$ & $\begin{array}{l}\text { Pequenos derramamentos } \\
\text { de óleo devidos a } \\
\text { movimentos operacionais } \\
\left(10^{4}-10^{5} €\right)\end{array}$ & $\begin{array}{l}\text { Má publicidade local para } \\
\text { o porto }\left(10^{4}-10^{5} €\right)\end{array}$ & 2 \\
\hline Sérias & $\begin{array}{c}\text { Múltiplas lesões } \\
\text { ligeiras ou uma } \\
\text { única lesão } \\
\text { grave }\end{array}$ & $10^{2}-10^{3} €$ & $\begin{array}{l}\text { Embarcação passível de } \\
\text { ser limitada a uma zona } \\
\text { de segurança do porto } \\
\qquad\left(10^{5}-10^{6} €\right)\end{array}$ & $\begin{array}{c}\text { Má publicidade } \\
\text { generalizada, restrições } \\
\text { à navegação, possivel } \\
\text { encerramento parcial } \\
\left(10^{5}-10^{6} €\right)\end{array}$ & 5 \\
\hline Muito Sérias & $\begin{array}{l}\text { Muitas lesões } \\
\text { graves ou uma } \\
\text { perda de vida }\end{array}$ & $10^{3}-10^{4} €$ & $\begin{array}{l}\text { Esperados episódios de } \\
\text { poluição dentro e fora do } \\
\text { porto com potenciais } \\
\text { perdas irrecuperáveis } \\
\text { para o ambiente } \\
\left(10^{6}-10^{7} €\right)\end{array}$ & $\begin{array}{l}\text { Má publicidade a nível } \\
\text { nacional, encerramento } \\
\text { temporário do canal de } \\
\text { navegação afectando } \\
\text { navegação no porto } \\
\text { durante vários dias }\left(10^{6} \text { - }\right. \\
\left.10^{7} €\right)\end{array}$ & 10 \\
\hline Catastróficas & $\begin{array}{l}\text { Muitas perdas } \\
\text { de vida }\end{array}$ & $>10^{4} €$ & $\begin{array}{c}\text { Derrame de petróleo } \\
\text { grave, necessária ajuda } \\
\text { internacional, } \\
\text { contaminação séria, } \\
\text { perdas irrecuperáveis } \\
\text { para o ambiente }\left(>10^{7} €\right)\end{array}$ & $\begin{array}{l}\text { Má publicidade } \\
\text { Internacional, } \\
\text { encerramento do porto, } \\
\text { navegação interrompida } \\
\text { durante um longo } \\
\text { período, perda } \\
\text { considerável de comércio } \\
\left(>10^{7} €\right)\end{array}$ & 25 \\
\hline
\end{tabular}


- Definição do regime de agitação marítima no centro de gravidade de cada troço da trajectória do navio;

- Cálculo do movimento vertical de um ponto seleccionado do navio. Neste trabalho, utilizase o modelo numérico WAMIT, (J.N. Newman e P.D. Sclavounos, 1988), para a interacção entre o corpo flutuante e a agitação marítima;

- Cálculo do grau de risco de excedência do limiar préestabelecido para os movimentos verticais do navio através da análise quantitativa da probabilidade de excedência desse limiar e de uma análise qualitativa das respectivas consequências.

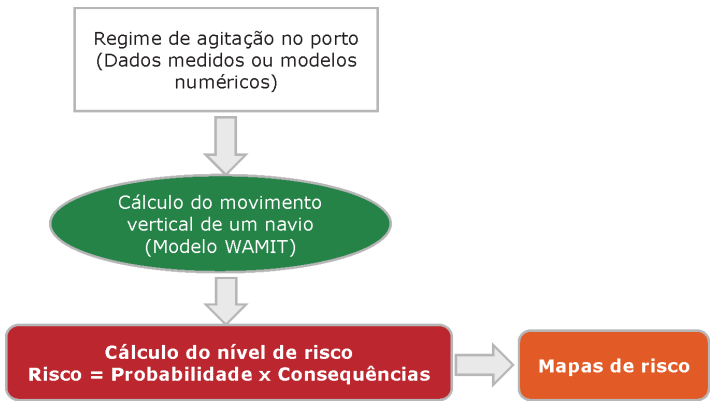

Figura 1 - Metodologia de avaliação do risco.

O sistema GUIOMAR foi utilizado, quer no estabelecimento dos regimes de agitação marítima (dentro e fora do porto), quer na aplicação da metodologia de avaliação de risco assim como na produção automática de mapas de risco.

As secções seguintes descrevem os procedimentos associados a cada um dos passos apresentados anteriormente.

\section{Regime de agitação marítima no interior do porto}

O cálculo do regime de agitação marítima no interior do porto, i.e., ao longo da trajectória do navio, tem como base:

a) As características da agitação marítima medidas in situ (o que não é normalmente de fácil de obtenção);

b) A transferência do regime de agitação ao largo para o interior do porto por intermédio de um ou mais modelos numéricos de propagação de ondas.

A utilização de um ou mais modelos numéricos de propagação de ondas depende das características da região em estudo (por exemplo, a dimensão do respectivo domínio de cálculo) e dos fenómenos envolvidos na propagação das ondas (refracção, difracção e rebentação). No presente trabalho utilizouse o modelo SWAN (N. Bools et al., 1999) e/ou o modelo DREAMS (C.J.E.M. FORTES, 2002).

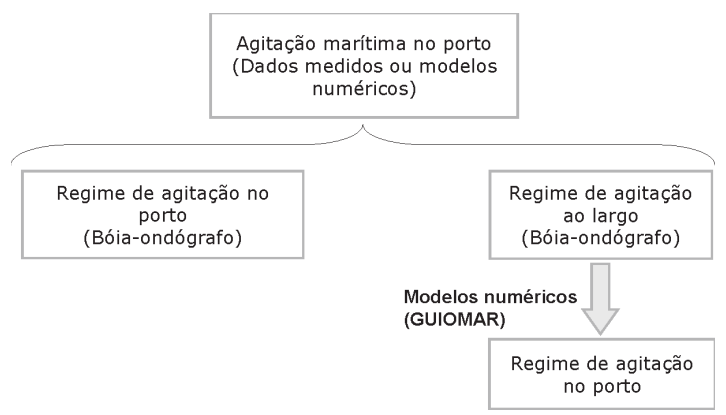

Figura 2 - Metodologia de cálculo da agitação marítima.

Cálculo dos movimentos do navio

Escolhido o navio cujo comportamento se pretende estudar, utiliza-se o modelo numérico WAMIT (J.N. NEWMAN e P.D. ScLAVounos, 1988) para determinar a resposta do navio, em termos da amplitude dos movimentos segundo cada um dos seis graus de liberdade do mesmo, Fig. 3, para uma gama de períodos de onda, profundidades e ângulos entre o eixo longitudinal do navio e o vector número de onda expectável na zona em estudo.

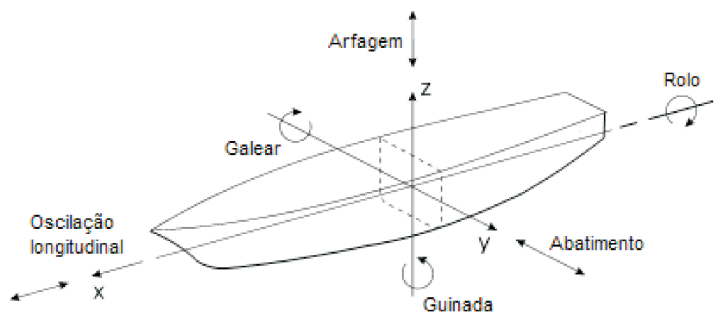

Figura 3 - Movimentos do navio considerado pelo modelo WAMIT.

Os movimentos, cujas amplitudes o modelo numérico WAMIT calcula, são os da origem do referencial baricêntrico do navio, mostrado na Fig. 3. Como tal, para se obter a amplitude do movimento vertical de qualquer ponto do navio, é necessário avaliar a componente vertical do produto externo do vector velocidade de rotação pelo vector de posição desse ponto do navio em relação à origem do referencial baricêntrico. Importa referir que as componentes do vector velocidade de rotação são grandezas complexas pois, para além do módulo da velocidade de rotação em torno de cada um dos eixos, importa conhecer também a fase da mesma velocidade.

No fim deste procedimento, resulta uma matriz de transferência que estima a amplitude da componente vertical do movimento de um ponto do navio a partir da profundidade da zona onde o navio se desloca, do período da onda e do ângulo entre o eixo longitudinal do navio e o vector número de onda da onda incidente no navio. 
$\mathrm{Na}$ abordagem preliminar que aqui se descreve, não se levou em conta a velocidade de avanço do navio, nem a irregularidade da agitação marítima incidente no navio, considerando para este caso a existência de ondas regulares. Importa referir também, que foi assumido que o contorno da bacia portuária está suficientemente afastada do navio para influenciar a resposta do mesmo.

Em resumo, em qualquer um dos baricentros dos troços em que se dividiu a zona passível de ocupação pelo navio ao longo da sua trajectória de entrada no porto, basta conhecer a batimetria do porto nesse ponto, as características da ondulação aí incidente (altura, período e direcção) e o rumo do navio. Com essa informação (a altura da onda excluída) determina-se, com a matriz de transferência, a amplitude do movimento do ponto de interesse para uma onda incidente com amplitude unitária. Como se considera linear a interacção entre o navio e as ondas, basta multiplicar o valor da amplitude do movimento resultante da matriz de transferência pela amplitude correspondente da onda incidente para se obter a amplitude do movimento para a dada onda.

\section{Avaliação do risco}

A avaliação do risco para a navegação de um navio tem em conta apenas o movimento vertical de um ponto seleccionado do navio obtido dos resultados do modelo WAMIT. Desta forma, determina-se qualitativamente o risco para a navegação associado à excedência de um limiar pré-estabelecido para a amplitude daquele movimento em cada um dos troços em que se divide a área do porto varrida pelo navio na sua trajectória de entrada no porto.

A metodologia de avaliação de risco para uma dada zona portuária implementada no sistema GUIOMAR baseia-se em cinco etapas principais (Fig. 4):

- Definição dos troços onde serão calculados os movimentos de um ponto do navio a partir dos resultados do modelo WAMIT (tal implica o conhecimento das condições de agitação marítima nos baricentros desses troços);

- Definição do limiar aceitável para a amplitude do movimento vertical do navio em cada um daqueles troços;

- Desenvolvimento de uma tabela de probabilidade de ocorrência de movimentos verticais de um navio que excedem os limiares pré-estabelecidos e determinação do grau de probabilidade de ocorrência para os vários troços em estudo;

- Desenvolvimento de uma tabela de consequências da ocorrência de movimentos verticais excessivos de um navio e determinação do grau de consequências para os vários troços em estudo;
- Multiplicação dos valores do grau de probabilidade de ocorrência pelos valores respectivos do grau de consequências para obter o grau de risco associado ao limiar pré-estabelecido para cada troço.

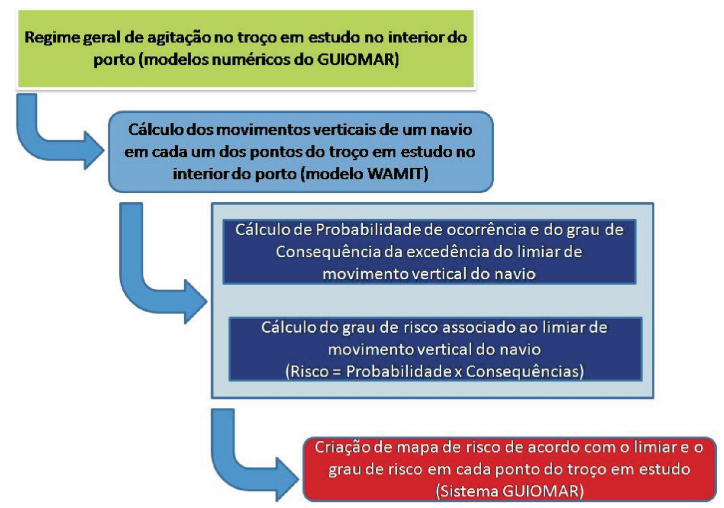

Figura 4 - Metodologia para a avaliação de risco numa zona portuária.

\section{Sistema GUIOMAR}

O sistema GUIOMAR (A.C. Zózimo e C.J.E.M. Fortes., 2007) (A.C. Zózımo et al., 2008) (D.R.C.B. Neves et al., 2009A,B) é um sistema integrado para a modelação numérica em engenharia costeira e portuária, desenvolvido em linguagem de programação VBA (Visual Basic for Applications), num sistema de informação geográfica (SIG). É composto por três componentes principais:

- Um sistema de informação geográfica, e neste caso, no software comercial de SIG o ArcGIS'M;

- Um conjunto de seis módulos correspondentes a diferentes modelos de propagação e deformação da agitação marítima com diferentes domínios de aplicação;

- Uma interface gráfica (GUI), desenvolvida em linguagem de programação VBA, responsável pela ligação entre o software SIG e os modelos numéricos de propagação de ondas, possibilitando: a) a execução dos modelos numéricos; b) efectuar o pré e pós processamento de dados e de resultados dos modelos numéricos; e c) a utilização de funcionalidades existentes no software SIG , tais como a análise e a visualização de dados e resultados, tanto em 2D como em 3D.

Presentemente, o sistema GUIOMAR alberga seis módulos/modelos: três modelos baseados na equação de declive suave, DREAMS (C.J.E.M. FoRTES, 2002), REF/DIF 1 (R. DalRYMPLE e J.T. KIRBY, 1991), REF/DIF S (J.T. KIRBY e H. OzKAN, 1994); dois modelos baseados na equação de Bousssinesq, FUNWAVE 1D e 2D (J.T. KIRBY et al., 1998); e o modelo espectral de geração, propagação e dissipação da agitação marítima SWAN (N. Bools et al., 1999). O programa GMALHA (L.V. PINHEIRO et al., 2006) constitui 
também um módulo, sendo responsável pela geração de malhas computacionais não estruturadas para os modelos numéricos e pode ser aplicado independentemente ou de forma acoplada.

A natureza modular do sistema permite ao programador efectuar de forma fácil os melhoramentos e actualizações, tal como a inclusão de novos modelos numéricos com capacidades superiores e que simulem diferentes processos físicos. O sistema GUIOMAR caracteriza-se pela implementação, em termos de programação do sistema, de vários procedimentos automáticos para reduzir a ocorrência de erros humanos e para acelerar a familiarização do utilizador com os procedimentos de operação dos modelos numéricos.

0 mais recente desenvolvimento no sistema GUIOMAR envolve a construção de um novo módulo com o objectivo de produzir automaticamente mapas de risco de acordo com a metodologia apresentada na secção da metodologia de avaliação de risco. A Fig. 5 representa a barra de ferramentas do sistema GUIOMAR através da qual é possível aceder à área de produção de mapas de risco.

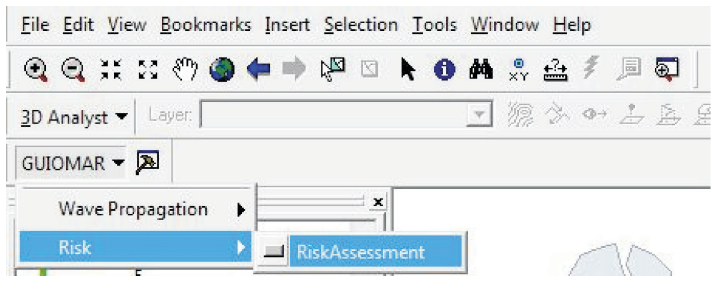

Figura 5 - Barra de ferramentas GUIOMAR com opção para avaliação de risco.

Depois de accionar a tecla "Risk assessment", surge uma nova interface gráfica no GUIOMAR (Fig. 6) capaz de, em 3 passos, construir o mapa de risco associado a movimentos verticais do navio e, se desejável, exportar os resultados de forma visível no Google Earth ${ }^{T M}$ :

- “Load site image" - carrega a imagem georeferenciada do local para o ambiente de trabalho do ArcGis $^{T M}$;

- "Load local risk Assessment" - Lê o ficheiro (.txt) com o grau de risco associado a cada ponto em estudo e transforma em shapefile no $\operatorname{ArcGis}^{T M}$, atribuindo uma bandeira de determinada cor de acordo com o valor do grau de risco associado a cada ponto;

- "Export to Google Earth" - Exporta os pontos e as cores das respectivas bandeiras que sinalizam o risco em cada ponto num ficheiro (.kmz) para abrir em Google Earth $^{\text {TM }}$.

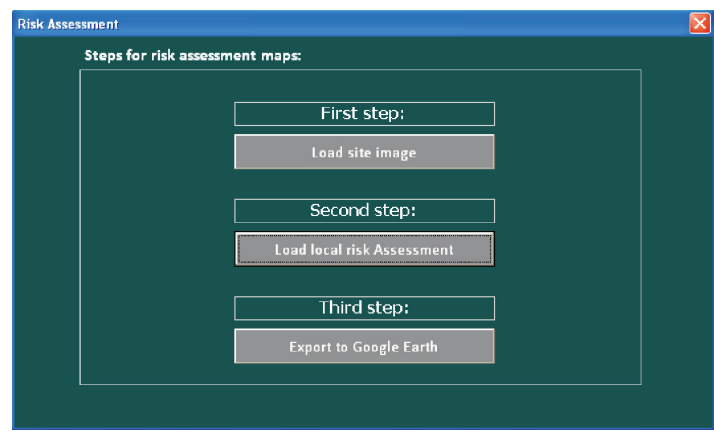

Figura 6 - Interface GUIOMAR para a criação dos mapas de risco para a navegação.

O ficheiro criado durante o cálculo do grau de risco conterá a localização geográfica, em coordenadas militares, de cada ponto em estudo e o valor correspondente do grau de risco.

\section{CASO DE ESTUDO}

\section{Caracterização Geral}

Foi no porto de Sines que foram testados os novos desenvolvimentos do sistema GUIOMAR. O porto de Sines, localizado a $37^{\circ} 57^{\prime} \mathrm{N}$ e $08^{\circ} 53^{\prime} \mathrm{W}$, é uma das principais entradas comerciais e económicas da Península Ibérica, considerando-se um porto de extrema importância geográfica e estratégica para Portugal e Espanha, Fig. 7.

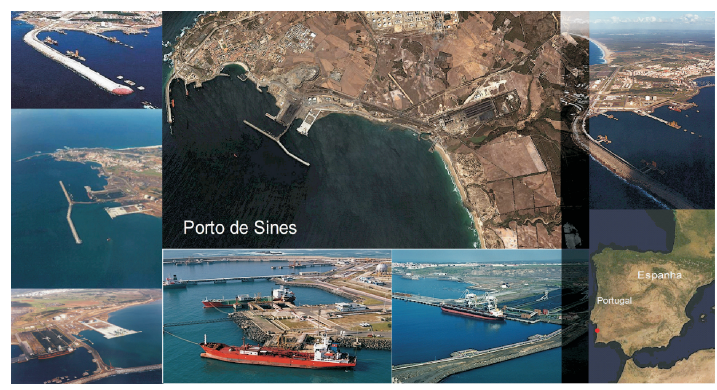

Figura 7 - Porto de Sines.

Neste momento, o complexo do porto de Sines é composto por várias infra-estruturas marítimas para abrigo e acostagem, tornando-o num dos poucos portos europeus de águas profundas que permitem a acostagem de navios de grande porte. Os elevados valores anuais de movimentação de mercadorias transformaram o porto de Sines no primeiro porto nacional em tonelagem anual movimentada.

Uma vez que se pretende avaliar o risco associado aos movimentos verticais de um navio ao longo da sua trajectória de entrada no porto de Sines e como esses movimentos são causados pela agitação marítima, 
apresenta-se a caracterização da agitação marítima ao longo dessa trajectória, na zona de entrada do porto e no interior do porto, bem como a resposta à acção das ondas do navio seleccionado.

\section{Cálculo do Regime de Agitação Marítima}

Para a caracterização da agitação marítima no local em estudo, recorreu-se aos dados de agitação marítima da bóia-ondógrafo designada por "Sines 1D", localizada ao largo do porto de Sines ( $37^{\circ} 55^{\prime} \mathrm{N}$ e $\left.08^{\circ} 55^{\prime} \mathrm{W}\right)$. O conjunto de dados utilizados perfaz um total de 32807 registos obtidos entre Maio de 1988 e Dezembro de 2002, (L.V. PinheIro et al., 2004).

Estes valores foram transferidos para o interior do porto com recurso a dois modelos de propagação e deformação da agitação marítima. Com efeito, dados os diferentes domínios de aplicabilidade de cada modelo, para a zona marítima do porto de Sines houve a necessidade de acoplar dois modelos numéricos, Fig. 8: o modelo SWAN e o modelo DREAMS. 0 modelo SWAN permite a propagação e deformação das ondas desde águas profundas até junto à entrada do porto, mas não pode ser utilizado em zonas confinadas, e o modelo DREAMS permite a simulação da agitação marítima na zona interior do porto (dado o esforço computacional envolvido não pode ser aplicado a grandes áreas de cálculo).

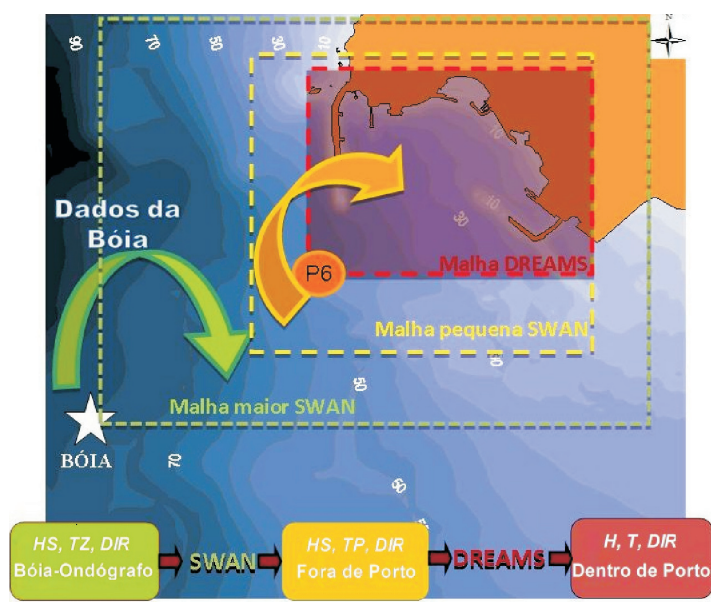

Figura 8 - Metodologia para a transferência do regime de agitação marítima do largo (bóia-ondógrafo) para o interior do porto.

Os dados obtidos na bóia são transferidos os registos da bóia para os pontos no interior do porto, obtendose o regime de agitação marítima em cada ponto. Os pontos definidos para a obtenção do regime de agitação no interior do porto através dos cálculos com o modelo DREAMS foram os pontos A, B, C, D, E, F e G (Fig. 9):

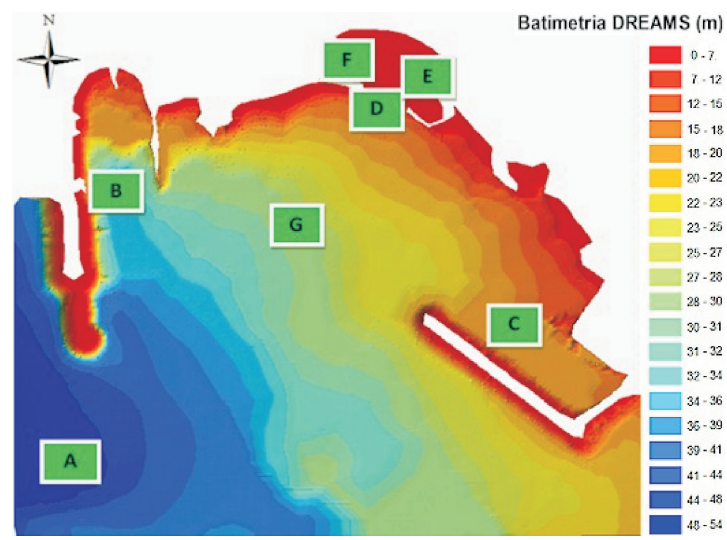

Figura 9 - Batimetria com a localização dos pontos A a G no interior do porto.

A título de exemplo, mostram-se na Fig. 10 para o ponto A o histograma geral HS-DIR, onde cada gráfico inclui as respectivas distribuições marginais e rosa de direcções.

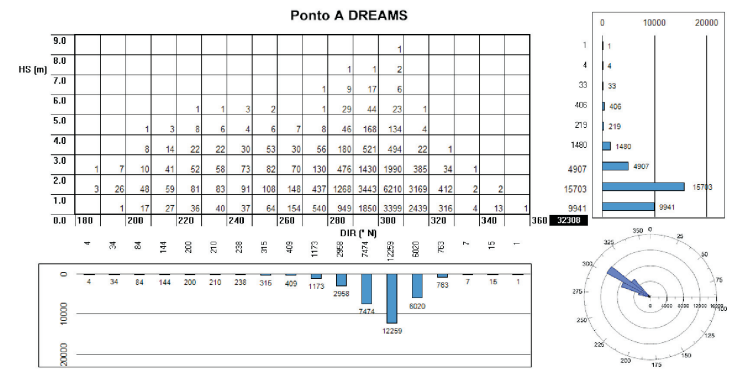

Figura 10 - Regime geral no ponto A: Histograma geral conjunto (HS, DIR) das ocorrências e respectivas distribuições marginais e rosa de direcções.

\section{Cálculo dos Movimentos do Navio}

No presente trabalho, foi determinada a resposta de um navio em termos de amplitude da oscilação vertical de um ponto seleccionado do cada navio, para diversos valores de períodos de onda regular incidente e para 25 valores do ângulo entre o eixo longitudinal do navio e a direcção de propagação da onda e para 7 valores da razão calado/profundidade, entre 1.1 e 5.0. Para os cálculos foi definido $1.00 \mathrm{~m}$ de altura da onda incidente.

O navio apresenta um deslocamento de $38.9 \mathrm{~m}^{3}$, comprimento de $12.00 \mathrm{~m}$, boca de $4.42 \mathrm{~m}$ e calado de $1.00 \mathrm{~m}$. A superfície molhada do casco foi discretizada com 534 painéis. Este navio pretende representar uma das traineiras que frequentam o porto de pesca. Os centros de gravidades dos troços em que se dividiu o percurso de, são os pontos P6, A, G, D e F. Para este navio os períodos da ondulação incidente variaram entre $1 \mathrm{~s}$ e $19 \mathrm{~s}$ com um intervalo de $1 \mathrm{~s}$.

Na Fig. 11 apresenta-se, para os valores da razão calado/ profundidade, a evolução da altura do movimento vertical de um ponto do navio $(-6.14,-1.19,0.00)$ 
(valores em metros), ponto este considerado para os cálculos no caso de estudo, com o período da onda incidente no navio quando o ângulo entre a onda e o eixo longitudinal do navio é igual a $30^{\circ}$. Importa referir que, embora não ilustrado neste artigo, se verificou serem as ondas quase de través (ângulos entre a direcção da onda incidente no navio e o eixo longitudinal do mesmo entre $45^{\circ}$ e $135^{\circ}$ ou entre $225^{\circ}$ e $315^{\circ}$ ) aquelas que produziam maiores alturas do movimento vertical no ponto.

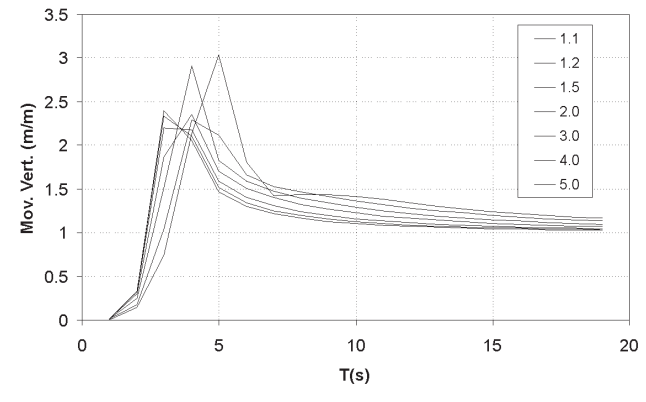

Figura 11 - Variação da altura do movimento vertical no ponto $(-6.14,-1.19,0.00)$ com o período da onda incidente e a razão calado/profundidade. Ângulo entre o eixo longitudinal do navio e direcção da onda incidente igual a $30^{\circ}$.

Interessante na figura é a resposta apreciável para valores baixos do período da onda incidente no navio ( $3 \mathrm{~s}$ a $5 \mathrm{~s}$ ) e o acompanhar da onda pelo navio para os períodos mais elevados (acima de $15 \mathrm{~s}$ ). Embora os valores apresentados na figura possam parecer exagerados, ultrapassando em alguns casos metade da profundidade da zona onde o navio está, importa não esquecer que estes são valores para uma onda cuja altura é $1 \mathrm{~m}$. Ora, para alguns dos valores de profundidade considerados, isso seria uma altura de onda exagerada, senão impossível. Além disso, para este navio cujo calado é $1 \mathrm{~m}$, a viagem de entrada do navio no porto de Sines é sempre realizada sobre regiões cuja profundidade ultrapassa cinco vezes o calado do navio, pelo que a curva de resposta do navio mais utilizada neste estudo é a mais baixa da figura.

Determinada a função resposta para as gamas de profundidades, períodos e ângulos já mencionados, procedeu-se ao cálculo da função resposta em cada um dos pontos do QuAdro V para o navio considerado e atendendo ao percurso definido. Como pode observar-se no referido quadro, o percurso determinou o ângulo a considerar entre o eixo longitudinal do navio e a direcção de propagação da onda.

Quadro V - Profundidades e rumos do navio nos baricentros dos troços em que foi dividido o percurso de entrada estudado

\begin{tabular}{|c|c|c|c|c|c|}
\cline { 2 - 6 } \multicolumn{1}{c|}{} & P6 & A & G & D & F \\
\hline Prof. (m) & 47.12 & 52.05 & 27.32 & 11.41 & 7.00 \\
\hline Rumo navio $\left(^{\circ}\right)$ & 90 & 45 & 30 & 0 & 315 \\
\hline
\end{tabular}

Uma vez definida a função resposta cada um dos pontos, para o cálculo da altura do movimento vertical do navio no troço da zona varrida na sua trajectória de entrada no porto, apenas se torna necessário conhecer os resultados de agitação marítima obtidos com os modelos numéricos SWAN e DREAMS nos mesmos pontos, isto é, os baricentros do troço em estudo: pontos P6, A, G, D e F (Fig. 12).
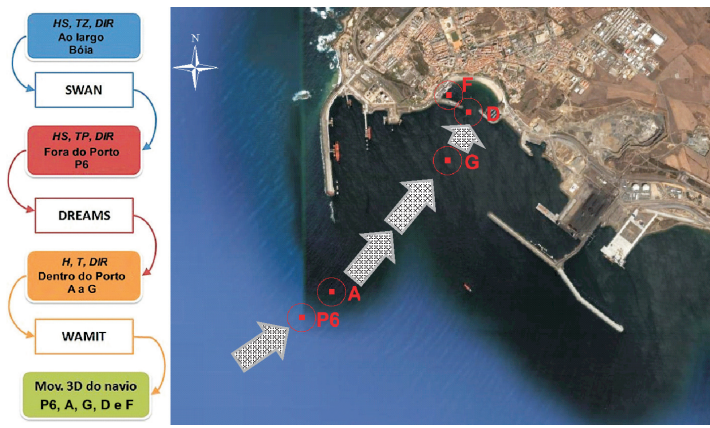

Figura 12 - Esquema de modelação para cálculo dos movimentos do navio e localização dos pontos onde foi aplicado o modelo WAMIT (imagem Google Earth ${ }^{T M}$ ).

\section{Avaliação de risco}

Para ilustrar e testar a metodologia de avaliação de risco apresentada anteriormente, foram definidos, a título de exemplo, dois limiares de $0.5 \mathrm{~m}$ e $1 \mathrm{~m}$ para a amplitude do movimento vertical do navio considerado e avaliado o risco dos dois limiares serem excedidos, considerando a probabilidade de ocorrência e as consequências de tais eventos nos pontos do percurso em estudo: P6, A, G, D e F (QUADRO VI).

O QuAdRo VI mostra que existem de facto algumas diferenças quando comparando os dois limiares de excedência de movimento vertical do navio. Verificase que a probabilidade de ocorrência da excedência do limiar de $0.5 \mathrm{~m}$ é bastante maior que a do limiar de 1 $\mathrm{m}$ sobretudo para os pontos mais interiores do porto de Sines, o que em termos objectivos torna o grau de risco obrigatóriamente inferior para o caso de exedência do limiar de $1 \mathrm{~m}$ devido ao valor mais baixo de grau concedido à probabilidade de ocorrência. No caso da avaliação qualitativa das consequências foram admitidas consequências mais gravosas (de grau superior) no caso da excedência do limiar de $1 \mathrm{~m}$, o que conduz obrigatóriamente a um aumento do grau de risco. Contudo importa dizer que é necessária uma abordagem mais precisa da avaliação do grau das consequências de forma a tornar a avaliação do risco o mais aproximada possível da realidade, os valores do grau de consequências aqui apresentados tiveram sobretudo um papel de teste da metodologia e dos procedimentos automáticos criados no decorrer do presente trabalho. Em termos gerais os 
resultados aqui apresentados mostram, como seria de esperar, e visto que o grau de consequências é superior ao grau de probabilidade de ocorrência da exedência do limiar, um risco mais elevado para a excedência 1 $\mathrm{m}$ porque $\mathrm{o}$ grau de consequências da sua excedência é mais elevado do que no caso da excedência do limiar de $0.5 \mathrm{~m}$.

Na Fig. 13 apresentam-se os mapas de risco criados a partir do módulo de construção de mapas de risco do sistema integrado GUIOMAR para o caso considerado. Nesta figura, a cor das bandeiras representa a cor associada a cada grau de risco (QUADRo VI).

\section{Conclusão}

Neste artigo, apresentaram-se os desenvolvimentos recentes no sistema GUIOMAR que consistiram num conjunto de procedimentos automáticos de implementação de uma metodologia de avaliação do risco para a navegação portuária. Recorrendo a dados da bóia-ondógrafo situada ao largo do porto de Sines e aos modelos SWAN e DREAMS, foi definido o regime de agitação marítima em vários pontos no interior do porto. Para verificar o efeito da agitação marítima num navio seleccionado foi utilizado o modelo WAMIT. Foi implementado um procedimento automático de avaliação do risco considerando que o grau de risco é o produto do grau atribuído à probabilidade de ocorrência de movimentos verticais de um navio que excedem limiares pré-estabelecidos pelo grau atribuído às consequências dessa excedência.

Este aperfeiçoamento do sistema GUIOMAR é um passo fundamental na implementação de uma metodologia de avaliação do risco para a navegação em zonas portuárias

Quadro VI - Avaliação do risco de ocorrência de movimentos verticais de dois navios que excedem os limiares pré-estabelecidos de $0.5 \mathrm{~m}$ e de 1

\begin{tabular}{|c|c|c|c|c|c|c|}
\hline & \multicolumn{3}{|c|}{ Limiar de $1 \mathrm{~m}$} & \multicolumn{3}{|c|}{ Limiar de $0.5 \mathrm{~m}$} \\
\hline Pontos & Probabilidade & Consequências & Risco & Probabilidade & Consequências & Risco \\
\hline \multirow{2}{*}{ P6 } & Frequente & Reduzidas & Aceitável & Frequente & Insignificantes & Aceitável \\
\hline & (> 50\%) Grau 5 & Grau 2 & Grau 10 & (> 50\%) Grau 5 & Grau 1 & Grau 5 \\
\hline \multirow{2}{*}{ A } & & Sérias & Indesejável & Frequente & Reduzidas & Aceitável \\
\hline & (> 50\%) Grau 5 & Grau 5 & Grau 25 & (> 50\%) Grau 5 & Grau 2 & Grau 10 \\
\hline \multirow{2}{*}{ G } & Raro & Muito sérias & Indesejável & Provável & Sérias & Indesejável \\
\hline & (1-10\%) Grau 2 & Grau 10 & Grau 20 & (25-50\%) Grau 4 & Grau 5 & Grau 20 \\
\hline \multirow{2}{*}{ D } & Raro & Catastróficas & Inaceitável & Ocasional & Muito sérias & Indesejável \\
\hline & $(1-10 \%)$ Grau 2 & Grau 25 & Grau 50 & $(10-25 \%)$ Grau 3 & Grau 10 & Grau 30 \\
\hline \multirow{2}{*}{$F$} & Improvável & Catastróficas & Indesejável & Improvável & Catastróficas & Indesejável \\
\hline & $(<1 \%)$ Grau 1 & Grau 25 & Grau 25 & $(<1 \%)$ Grau 1 & Grau 25 & Grau 25 \\
\hline
\end{tabular}
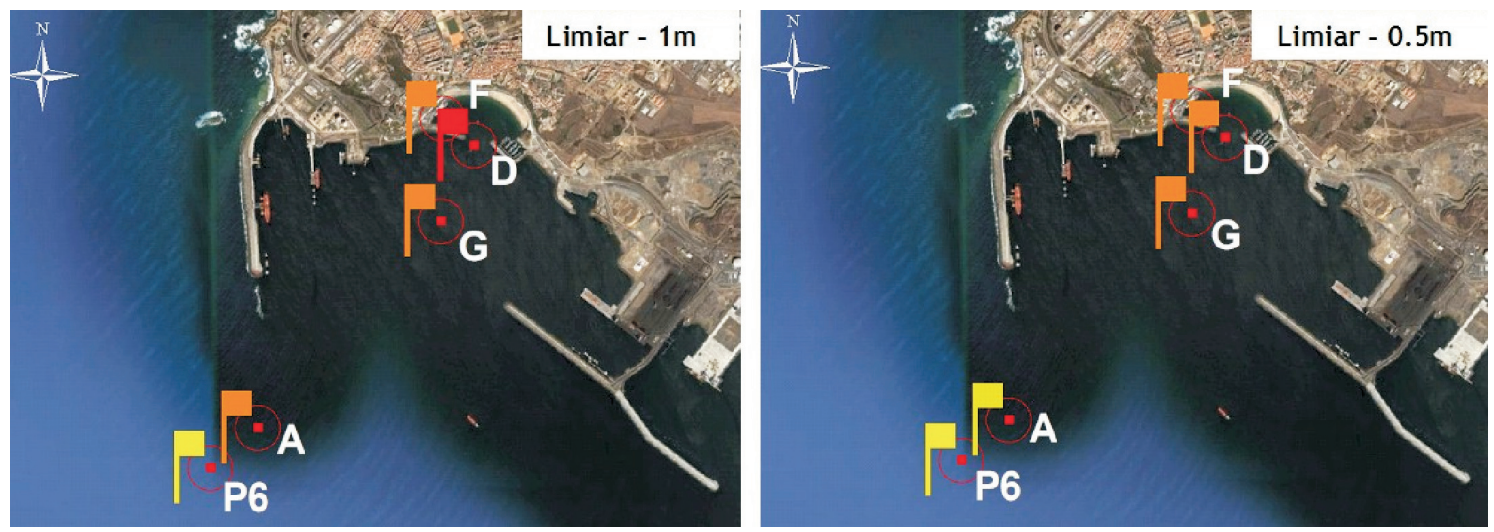

Figura 13 - Mapas de risco para a navegação no Porto de Sines (pontos P6, A, G, D e F), representativos do percurso considerado, relativamente a movimentos verticais que excedem $1.0 \mathrm{~m}$ e $0.5 \mathrm{~m}$. 
recorrendo à simulação de cenários com base em dados reais de agitação marítima.

No teste desta nova funcionalidade com o exemplo do porto de Sines, confirmou-se que o sistema GUIOMAR permite a realização de estudos de forma interactiva e amigável. Um sistema com a natureza do acima descrito constitui pois uma ferramenta importante para os estudos de segurança elaborados pelas autoridades portuárias.

A melhoria do sistema GUIOMAR constitui uma aposta de futuro, podendo-se referir a extensão do módulo construído no presente trabalho para a avaliação do risco de inundação e galgamento de estruturas portuárias ou até de operações de acostagem, carga e descarga de navios. Também está previsto o acoplamento entre os outros modelos que constituem o pacote de calculo numérico do sistema GUIOMAR, incluindo a verificação com dados reais, e a melhoria da visualização automática e interactiva dos dados e resultados dos modelos e dos mapas de risco.

\section{Agradecimentos}

Os autores agradecem à Fundação para a Ciência e a Tecnologia (FCT) o financiamento concedido através dos projectos PTDC/AMB/67450/2006, PTDC/ ECM/67411/2006 e PTDC/ECM/73145/2006.

\section{Referências bibliográficas}

BoolJ, N.; RIS, R.C.; HolthuIJSEn, L.H. (1999). "A thirdgeneration wave model for coastal regions, Part I, Model description and validation." J. Geophysical Research, 104, C4, pp. 7649-7666.

DalRymple, R.A.; KIRBY, J.T. (1991). “REF/DIF 1. Version 2.3 Documentation Manual. Combined Refraction/ Diffraction Model." CACR Report n. ${ }^{\circ}$ 91-2, University of Delaware, Janeiro.

ForTES, C.J.E.M. (2002). Transformações não lineares de ondas em zonas portuárias. Análise pelo método dos elementos finitos. Tese de doutoramento. Engenharia Mecânica, IST.

Kirby, J.T.; Ozkahn, H.T. (1994). "REF/DIF S Version 1.1 Documentation and Users Manual." CACR Report n. ${ }^{\circ}$ 94-04, University of Delaware.

Kirby, J.T.; WEI, G.; ChEN, Q.; KennedY, A.B.; Dalrymple, R.A. (1998). "FUNWAVE 1.0 - Fully Nonlinear
Boussinesq Wave Model Documentation and User's Manual." Research Report No.CACR-98-06, Center for Applied Coastal Research, University of Delaware.

Neves, D.R.C.B.; Zózimo, A.C.; Pinheiro, L.V.; Fortes, C.J.E.M. (2009A). "GUIOMAR: Geo(graphical) User Interface for cOastal and MARine Modeling. Supported Decision System." Journal of Coastal Research, SI 56 (Proc. 10th International Coastal Symposium), pp.15421546.

Neves, D.R.C.B; Zózimo, A.C.; Pinheiro, L.V.; Fortes, C.J. (2009B). "GUIOMAR: desenvolvimentos recentes e aplicação ao caso de Sines." Proc. $6^{a}$ s Jornadas Portuguesas de Engenharia Costeira e Portuária, Funchal, Outubro.

Newman, J.N.; Sclavounos, P.D. (1988). "The computation of wave loads on large offshore structures." Proc. Boss 88, Norway, pp. 1-19.

New Zealand Maritime Safety Authority (2004). New Zealand Port \& Harbour Marine Safety Code.

Pinheiro, L.V.; Coli, A.B.; Capitão, R.; Santos, J.A. (2004). "Caracterização da Agitação Marítima no Molhe oeste do Porto de Sines. Regime de extremos e eventos notáveis." Relatório 370/04- NPE, Lisboa, Novembro.

Pinheiro, L.V.; Fernandes, J.L.M.; Fortes, C.J.E.M. (2006). "Sobre um gerador de malhas de elementos finitos com densidade local condicionada à batimetria para modelos de propagação de ondas em zonas portuárias." Proc. I Conferência Nacional de Métodos Numéricos em Mecânica dos Fluidos e Termodinâmica, Lisboa, 8 e 9 de Junho.

Zózimo, A.C.; Fortes, C.J.E.M. (2007). “Estado Actual e Desenvolvimentos futuros do sistema integrado de apoio à tomada de decisão em engenharia costeira: GUIOMAR", Tecnologias da Água, n. ${ }^{\circ}$ 52, edição IV, Outubro/Dezembro, pp. 54-65.

Zózimo, A.C.; Fortes, C.J.E.M.; Neves, D.R.C.B. (2008). "GUIOMAR: Geographical User Interface for cOastal and MARine modeling. Recent developments and assessment of potential geographical errors", in Proc. Med Days of Coastal and Port Eng., Palermo, 7 a 9 de Outubro. 